\title{
EFFECT OF CONDENSABLE SPECIES ON PARTICULATE FOULING
}

\author{
K. K. Sathyanarayanarao Subbarao, C. C. M. Rindt and A. A. van Steenhoven \\ Faculty of Mechanical engineering, Energy technology group \\ Eindhoven university of technology, Post box 513, 5600MB, The Netherlands \\ k.kiran@tue.nl
}

\begin{abstract}
The flue gases emanating from the combustion of fuels or gasification process invariably comprises particulate matter and many chemical species in vapor form. The temperature of the flue gases gradually reduces when passing through different sections of heat exchanger like superheater, evaporator etc. If the temperature of the heat exchanger tube surface and the gas phase are favorable for condensation, the chemical species in the vapor form will condense on the particles and on the tube surface. The particle deposition behavior under these conditions is drastically different from the one observed in dry particulate fouling. In order to model the particle deposition under such circumstances, it is important to evaluate the criteria for particle adhesion to the surface. Impaction experiments of particles impacting a surface coated with a thin liquid film and particles which are coated with a liquid film impacting over a dry surface are performed to evaluate the limiting parameters under which a particle sticks to the surface without rebounding. The effects of liquid viscosity, liquid film thickness and interacting material properties are evaluated. The experimental results are compared to the results of existing models and a suitable model for fouling is proposed. Controlled fouling experiments are performed for varying liquid films coated over a deposition tube under various process conditions to mimic the condensation effects on fouling. The results are compared with the detailed impaction experiments.
\end{abstract}

\section{INTRODUCTION \\ Background}

The hot flue gas emanating from coal fired, co-fired and gasification process comprises several components in solid, semi-solid and vapor phases, depending on the position in the boiler section. Fouling occurs in the cooling section of the boiler where inorganic volatile substances condensate as compounds on the heat exchanger tube surface and forms a deposit layer along with the solid particulates. Due to the high temperatures, the particles are said to be in a state of semi molten phase and these may hit the tube and get stuck to the tubes. Over time, the deposit thickness grows and the outer temperature may reach the melting point of some of the constituents of the deposit. This provides active sites for other particles to deposit. If there are sufficiently large particles in the flow, the energy associated with them is higher. If these large particles impact on a tube surface with any pre-existing deposit layer, they can remove other particles. The structure of the deposited layers gives insight into the deposit forming dynamics and has been studied by various authors using advanced techniques like X-ray diffraction, electron probe X-ray micro analysis and Scanning Electron Microscopy (van Beek, 2001; Drift, 2004). A typical deposit structure collected from the super heater section in a biomass gasifier is shown in Figure 1. The deposit structure usually has two distinct layers: an inner layer and an outer layer. The inner layers were found to be hard, thick and compact, while the outer layers were coarser, with loosely bound particles (Drift, 2004). The detected distribution of elements over the local structure in the sample of the deposit indicated the formation of a melt in the deposit due to the presence of alkali substances. The structure of the layers differs in different cooler sections, like economizer and superheater. The economizer section is characterized by a thin powdery layer, while the superheater involves a two layered structure indicating a strong influence of temperature and condensation.

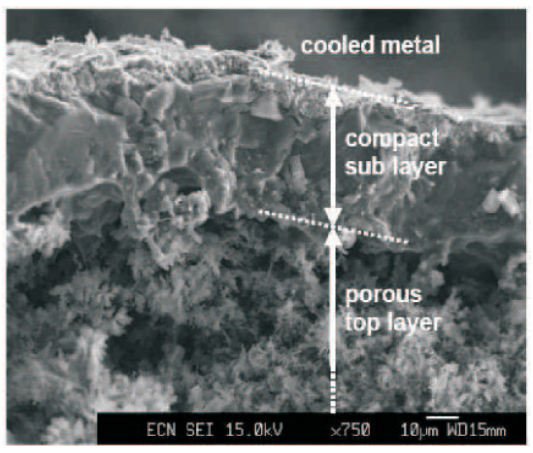

Figure 1. SEM picture of a fouled layer from superheater section of a biomass gasifier. (Drift, 2004).

Depending on the prevalent conditions in a certain section of the heat exchanger, condensation of vapors can occur in three variations:

a) Vapor may traverse the boundary layer and condense on the surface. 
b) Vapor may nucleate to form a fume and subsequently deposit by thermophoresis on the surface.

c) Vapors may heterogeneously condense on other particles and arrive at the surface.

A schematic representation of particle-surface interactions is shown in Figure 2.

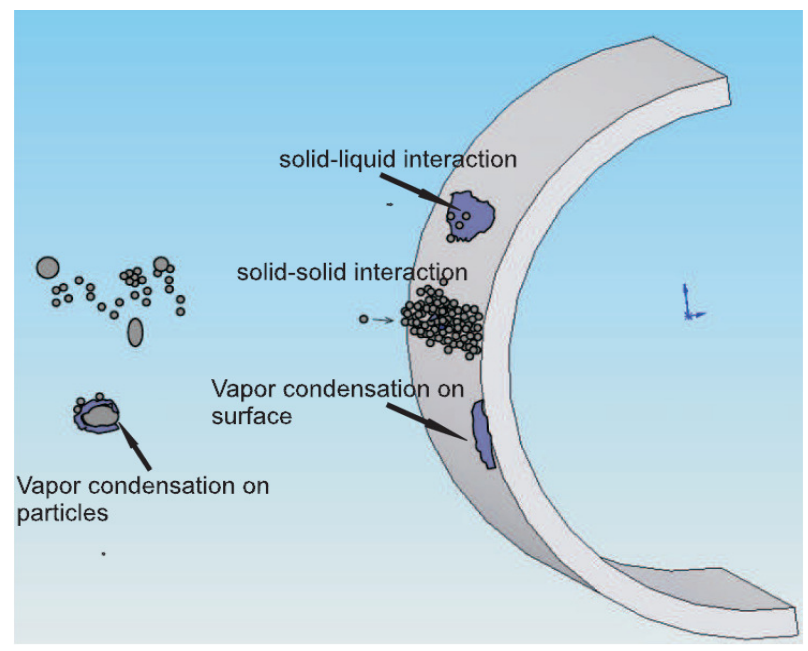

Figure 2. Schematic representation of deposition process.

\section{Modeling approaches}

The factors which determine whether a particle approaching a surface will eventually stick to the surface, rebound or remove other particles from the surface are plenty. The evaluation of the "sticking probability" under the influence of a thin liquid layer is still at large not answered. Basically three approaches are identified in the literature.

The first one corresponds to the melt fraction approach. In the presence of condensed alkali species, Rosner and Nagarajan (1992) proposed that the sticking probability depends on the amount of "glue" available for particle capture. The condensed alkali vapors act as the glue and are dependent on the melting behavior of the material on the deposit. It is reported that for alkali and alkali mixtures, a deposit becomes sticky when it contains between $10 \%$ and $70 \%$ melt (Isaak et al, 1986 and Tran, 2002). If the melt fraction is less than $10 \%$, it is treated as non sticky and for a melt fraction more than $70 \%$, it is treated as flowing liquid. The second approach is applicable for deposition at temperatures larger than $1000{ }^{\circ} \mathrm{C}$ and especially for slagging. The basis of this model lies in the rheological aspects of ash at high temperatures. The sticking probability is taken to be inversely proportional to the viscosity. A reference viscosity value is defined and viscosities lower than this value implies that the particles are perfectly sticky. For values greater than the reference viscosity, the particles are treated as non-sticky. The detailed explanation of this approach can be found in Bryers (1996) and Vargas (2001). A third approach is based on the critical velocity approach which is based on the concept of particle kinetics and the energy interactions involved in particle-wall and particle- particle collisions. The concept of critical velocity stems from the theory of contact mechanics.

The melt fraction approach is limited to high alkali condensations. However, in real systems, vapors of different chemicals might be present. The model predicts deposition for a melt fraction between $10 \%$ and $70 \%$ and does not explain dry particle deposition. As mentioned, the critical viscosity approach is valid for slagging conditions. Since condensation can occur in different sections and form thin films over the particle and the heat exchanger surface, the critical velocity approach seems to be most suitable to evaluate the sticking criteria. This approach however requires models to describe the amount of energy that is dissipated in the interstitial liquid layer during a particlesurface impaction process. A detailed literature survey on this topic directed to two models. Both models were compared with each other and it was found that the models have limited applicability.

\section{Aim of the present study}

The present research aims at understanding the outcome of a particle impacting a thin liquid coated surface at the fundamental level. This understanding is vital for numerical modeling of particle deposition. A condition for a particle to stick to the surface under the influence of interstitial liquid film is evaluated. Further, the aim is to understand the global effects of thin liquid films on particle deposition.

The theoretical development of the problem, basic impaction experiments and comparison to the results using literature models are discussed in the next few sections. Besides, deposition experiments under controlled conditions are performed to evaluate and relate the fundamental understanding to the real application of particle deposition. Initial results for particle deposition over a thin liquid coated tube are discussed.

\section{THEORY AND EXPERIMENTS - Part1 "Wet" Impaction}

Consider a particle impacting a substrate coated with a liquid film which is also referred to as "wet impaction". The process can be visualized in four different phases as proposed by Antonyuk et al (2009). The first phase represents the entry during which the particle penetrates the liquid layer and comes in contact with the solid surface. The second phase is similar to a dry impaction case which results in elastic plastic deformation process between the particle and the substrate. The third phase begins at the start of particle rebound until it reaches the liquid layer height. The fourth phase represents the liquid bridge formation and rupture of the bridge. Figure 3 shows the different phases schematically.

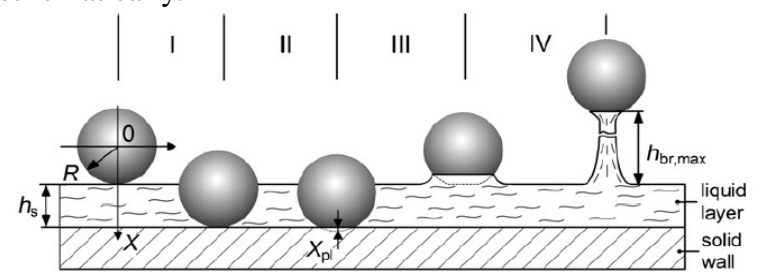

Figure 3. Schematic of different phases in a "wet" impaction process (Antonyuk, 2009). 
Figure 4 shows the snapshots of the process of particle impaction on a liquid coated surface as captured in high speed recording.

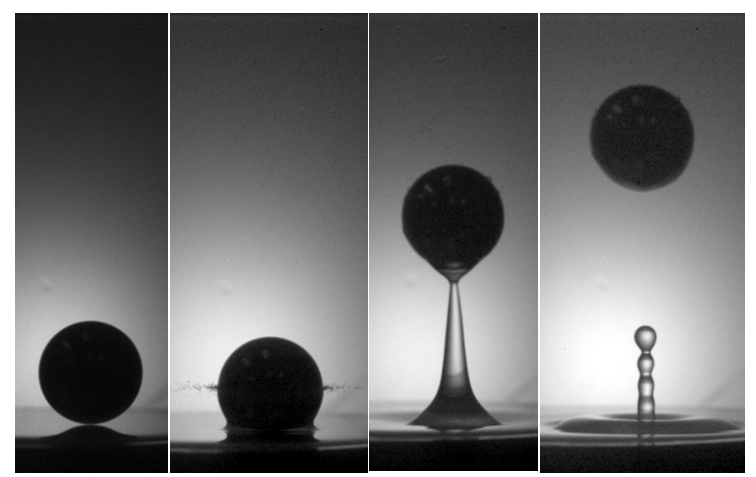

Figure 4. Snapshots of wet impaction process over water layer of $1 \mathrm{~mm}$ thickness.

Davis et al (1986) (2002) proposed a model describing the "wet" impaction according to which, the rebound characteristics of a particle impacting a liquid coated surface are given by the relation:

$$
\frac{v_{r}}{v_{o}}=e_{w e t}=e_{d r y}\left(1-\frac{S t_{c}}{S t}\right), S t>S t_{c}
$$

Here, $e_{w e t}$ is the co-efficient of restitution (COR) for the wet case, $e_{\text {dry }}$ the COR for dry impaction, $v_{r}$ is the rebound velocity, $v_{o}$ is the impact velocity, $S_{t c}$ is the critical Stokes number and $S t$ is the Stokes number.

The Stokes number is defined as:

$$
S t=\frac{m_{p} v_{o}}{6 \pi \mu_{l} r_{p}^{2}}
$$

Where, $m_{p}$ is the mass of the impacting particle, $\mu_{1}$ is the liquid layer viscosity and $r_{p}$ is the particle radius. The critical Stokes number is the maximum Stokes number above which particle starts to rebound and is given by the following relation:

$$
S t_{c}=0.40 \ln \left(\frac{1}{\varepsilon}\right)-0.20
$$

$\mathcal{E}$ is the elasticity parameter which provides a measure of tendency of the solids to deform and is given as:

$$
\varepsilon=\left(\frac{4 \theta \mu_{l} v_{o} r_{p}^{3 / 2}}{x_{o}^{5 / 2}}\right)
$$

Where, $x_{0}$ is the initial separation between the particle and the surface. $\theta$ is a function of Poisson's ratio and Young's moduli of the particle and substrate (Davis et al, 2002).

Thus, knowing the value of dry COR and the material properties of the particle and the substrate, the value for wet COR can be evaluated. The model was developed for particle-substrate undergoing purely elastic collisions and for perfectly hydro dynamically smooth surfaces. Further, the model is valid for a gap Reynolds number less than one. The gap Reynolds number is defined by the equation:

$$
\operatorname{Re}_{g a p}=\frac{\rho v_{o} h_{l}}{\mu_{l}}
$$

Where $\rho_{1}$ is the density, $\mu_{1}$ is the viscosity and $h_{l}$ is the thickness of the liquid layer.

Antonyuk et al (2009) proposed a model in which the effects of different forces acting on the particle are considered in detail. For a particle undergoing an impaction process as described in Figure 3, the kinematic equations that describe the relative motion of the particle is governed by Newton's law and can be expressed in the form:

$m_{p} \frac{d^{2} \vec{x}}{d t^{2}}=\vec{F}_{p, g}+\vec{F}_{t}+\vec{F}_{b}+\vec{F}_{D}+\vec{F}_{c}+\vec{F}_{v i s}+\vec{F}_{c a p}+\vec{F}_{l, g}$

The left hand side of equation 6 represents the mass and acceleration. The terms on the right side are the forces that act on the particle like: gravity, surface tension, buoyancy, drag, contact, viscous dissipation, capillary and liquid bridge forces. A detailed description of all the forces and equations can be found in Antonyuk et al (2009). The coefficient of restitution in this case is calculated using the relation:

$e=\sqrt{\frac{E_{k i n, R}}{E_{k i n, 0}}}=\sqrt{1-\frac{E_{\text {diss }, \text { tot }}}{E_{k i n, 0}}}=\frac{\left|v_{R}\right|}{v_{0}}$

The term $E_{\text {diss, tot }}$ refers to the energy dissipated in the overall process and $\mathrm{E}_{\mathrm{kin}}$ is the particle kinetic energy before the impact. $\mathrm{E}_{\text {diss, tot }}$ is evaluated using equation 5 .

To check the range of applicability of the available models, impaction experiments (similar to the experiments reported by Davis and Antonyuk) were performed and compared with the models. The following section describes the experimental setup, material and methods along with the comparison of the experimental results with the model predictions.

\section{Experimental setup}

Free fall experiments were conducted to evaluate the bouncing behavior of particles impacting a surface coated with a liquid film. The schematic of the experimental setup is shown in Figure 4. The experimental setup mainly consists of: a particle dropping head, target material, recording camera and corresponding lighting system.

Small particles in the range 1.5 to $5 \mathrm{~mm}$ in diameter were dropped using a perforated disc in housing. The perforated disc when rotated slowly allowed to particle to fall freely on the target at the same spot each time. Care was taken to turn the disc slowly so that the ball does not rotate while dropping down. A thick metallic plate with a reamed hole was used as a dropping mechanism for larger particles in the range 8 to $16 \mathrm{~mm}$. A spring loaded pin held the particle in place before dropping the particles. Marking dots were used on larger particles to ascertain the rotation effect and was 
found to be negligible. The target materials chosen were circular discs of 80 and $100 \mathrm{~mm}$ in diameter and $40 \mathrm{~mm}$ thick. Thickness of the target was chosen to avoid the effects of elastic wave propagation and vibration. A recessed pocket was provided on the top of the target material to act as a liquid reservoir. The depth of the pocket ranged from $0.15 \mathrm{~mm}$ up to $2 \mathrm{~mm}$ which corresponds to the liquid layer thickness.

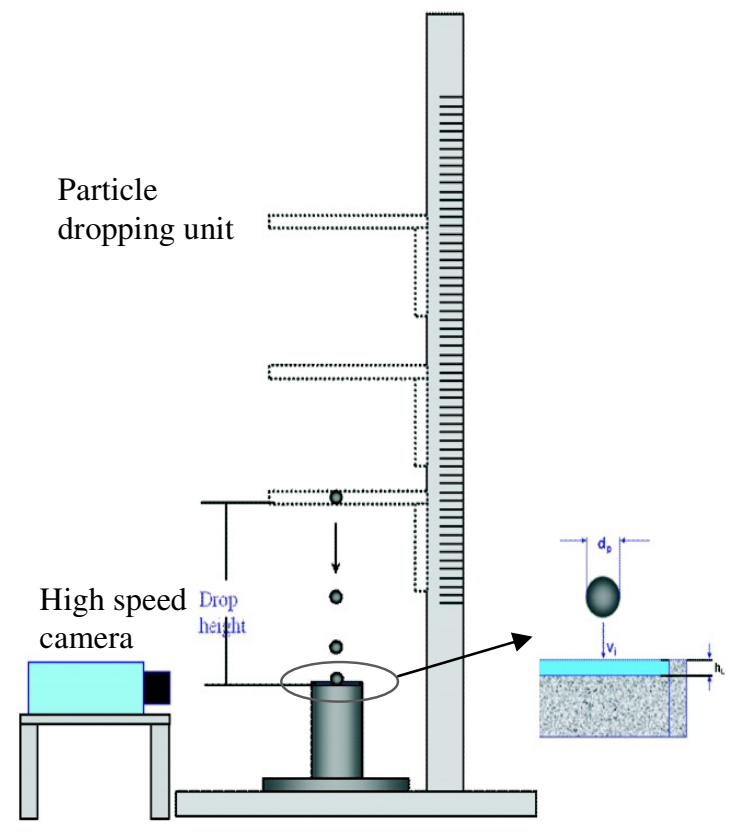

Figure 4. Schematic of the experimental setup used for the free fall experiments.

The surface of the pocket was made smooth with a emery paper and later polished with a diamond paste to get a smooth surface. Roughness measurements of the surface indicated an average roughness value in the range 1 to $3 \mu \mathrm{m}$ (RMS value). Glycerol, water, lubricating oils and an assortment of aqueous mixture of glycerol and water were used in the experiments. The viscosity of each liquid was measured before each experiment with a Rheometer [Rheometric Scientific TM ARES]. Glass, hardened steel (ball bearing), copper, brass, bronze, and acrylic particles of various sizes were used as the impacting particles. The dropping head and the base were leveled with a spirit level and digital level gauge before each experiment.

The impaction of small particles was measured with a camera of resolution 680x800 pixels [Jai CV M10]. The particle trajectory was illuminated with a laser sheet. The continuous laser was made intermittent using an optical chopper. The experimental methodology is similar to the one used by van Beek (2001) to measure dry impactions of particles. However, the present experiments were not done in a vacuum chamber as the particles size and density is large enough to neglect drag force induced by air. This procedure is consistent with the experiments reported by Davis and Antonyuk. This method allows the measurement of incident and rebound velocities, and hence to evaluate the coefficient of restitution. It will be shown later on, that the height to which the particle will rebound after impact is an important factor to evaluate the energy dissipated in the viscous layer. To measure to maximum rebound height and to achieve a range of Stokes numbers, particles of larger size were used (6 to $16 \mathrm{~mm}$ in diameter). For larger particles, the impact process was observed with a [Phantom V7] high speed camera. The recording rates ranged from 2 $\mathrm{kHz}$ to $15 \mathrm{kHz}$ based on the particles and drop velocity used. An LED light source was used to illuminate the region of interest from the rear and a thin diffuser plate was used to get a bright background. The choice of LED also helps in eliminating the changes in viscosity due to heat unlike other heat producing light sources. The particle trajectory was seen as a shadow [known as "shadowgraph technique"] which provides images with sharp edges of the particle which is essential for proper post processing and data analysis rather than direct particle illumination which results in reflection and noise. A calibration image was taken before each setting of the camera to calculate the velocity of the incoming and rebounding particle. The resolution of the camera changes with the recording rate (recording at lower rates can be made at higher resolutions and vice versa) and the field of view changes for different particle sizes. The overall error and uncertainty in the measurements and experimental process was found to be a maximum of $7 \%$. The velocity of the incoming and rebounding particle was measured by measuring the scaled distance traversed by the particle between each image and the frequency of recording.

\section{RESULTS (Part1: Comparison with the models)}

Figure 5 shows the experimental data (open symbols), model of Davis (M1: dotted lines) and model of Antonyuk (M2: dashed lines) for different liquid layer thicknesses and viscosities.

The model proposed by Davis et al captures the trend seen in the experimental results but over predicts the value for $\mathrm{e}_{\mathrm{wet}}$. Further, the changes in the value for $\mathrm{e}_{\mathrm{wet}}$ for varying liquid layer height are not captured. Figure $5 \mathrm{~b}$ and $5 \mathrm{c}$ for lower stokes number indicates that the model proposed by Antonyuk et al (2009) predicts rebound of particles for St> 45. However, the experimental data suggests that particle rebound occurs for St $>6$. Further the model proposed by Antonyuk et al is computationally intensive as all the different forces are to be calculated and it also seems that the viscous dissipation is over predicted resulting in prediction of particle sticking for up to $\mathrm{St}=45$ and no rebound at all for higher viscosity liquid film (Figure $5 \mathrm{~b}$ and 5c).

In short, the model of Davis is limited by only elastic deformation and over predicts the $\mathrm{e}_{\mathrm{wet}}$ values. The model of Antonyuk is complicated (though detailed) and the viscous dissipation is over predicted.

The need to evaluate the energy losses in terms of non dimensional numbers to obtain simple correlations seems to be a better option. The following section describes the methodology used to evaluate the energy balance of the wet impaction process. 

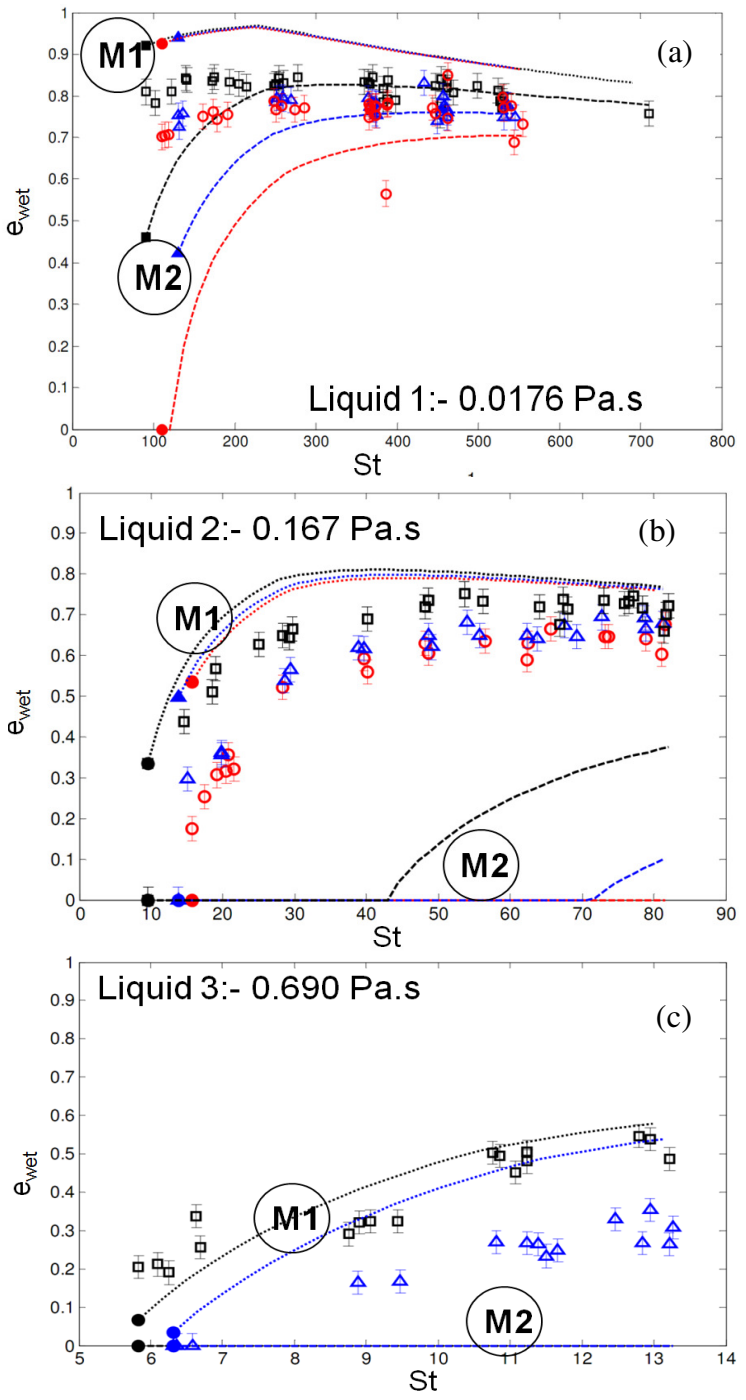

Figure 5. Comparison of wet COR $\left(\mathrm{e}_{\text {wet }}\right)$ with models. Open symbols represents experimental data. M1: model of Davis et al (1986) \{Dotted lines M2: model of Antonyuk et al (2009) \{Dashed lines Square: liquid layer thickness of $250 \mu \mathrm{m}$ Triangle: liquid layer thickness of $500 \mu \mathrm{m}$ Round: liquid layer thickness of $750 \mu \mathrm{m}$

\section{THEORY AND EXPERIMENTS - Part 2}

The experimental methodology and the results presented in part 1, where the particle incident and post impaction rebound velocities are used are inadequate to experimentally determine the energy dissipation of particles in various mechanisms. In order to understand the process in a better way, the following methodology was used:

\section{Energy Budget}

Particle-substrate interactions in the presence of a viscous interstitial fluid (liquid) can be visualized in two simple phases as shown in Figure 6

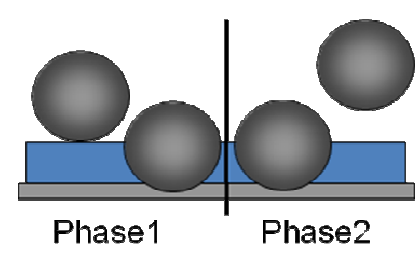

Figure 6. Schematic of particle impaction as a two step process

The first phase initiates when the particle comes in contact with the liquid layer and travels through the layer during which a certain part of the particle kinetic energy is dissipated in squeezing out the liquid from the gap between the particle and the substrate. During this process, the pressure that builds up is transmitted to the substrate. At this point, if the surface roughness of the particle and the substrate is "hydro dynamically smooth", it is reported that the particle will not touch the substrate (Happel and Brenner, 1965). However, in all practical situations, the particle comes in contact with the substrate owing to surface roughness and based on the kinetic energy, the particle might undergo pure elastic or elastic-plastic deformations. A good review and analysis of this is given by Zenit et al (1999). The first phase lasts till the particle just begins to rebound.

During the second phase, the particle rebounds from the surface forming a liquid bridge. The process of liquid bridge formation, rupture and droplet formation is not covered here and can be found elsewhere (Ennis et al, 1990, Simons and Seville, 1994). The particle rebounds to a maximum height where all of its kinetic energy in the rebound phase is converted to potential energy after which the particle falls down again.

An energy balance for such an interaction can be written as follows:

$\mathrm{E}_{\mathrm{kin}}-\mathrm{E}_{\mathrm{pot} 2}=\mathrm{E}_{\text {contact }}+\mathrm{E}_{\mathrm{viscous}}$

Where, $\mathrm{E}_{\mathrm{kin}}$ is the kinetic energy of the incoming particle given by; $E_{k i n}=0.5 \mathrm{~m}_{\mathrm{p}} \mathrm{v}_{\mathrm{o}}{ }^{2}, \mathrm{~m}_{\mathrm{p}}$ being the mass of the particle and $\mathrm{v}_{\mathrm{o}}$ is the incoming particle velocity.

$\mathrm{E}_{\mathrm{pot} 2}$ is the potential energy of the particle at the maximum height the particle reaches $\left(\mathrm{H}_{\text {rebound }}\right)$ after rebound and is given by the relation $\mathrm{E}_{\text {pot2 }}=\mathrm{m}_{\mathrm{p}} \mathrm{g} \mathrm{H}_{\text {rebound }}$.

$\mathrm{E}_{\text {contact }}$ is the energy dissipated in the contact phase of the particle with the substrate.

Case 1: For purely elastic contact, (neglecting generation of heat and sound), the energy dissipated as elastic strain energy is imparted back to the particle and hence $\mathrm{E}_{\text {contact }}$ for this case is zero.

Case 2: Elastic-plastic impact:

Considering a case where the particle is much harder than the substrate and the particle possesses enough inertia, the impact process results in an elastic-plastic interaction and an indentation/crater is formed over the soft substrate. By measuring the crater volume, the energy expended in the 
formation of the indentation can be evaluated (Roger and Reeds, 1984).

By measuring the impact velocity, rebound height and the crater size (in case of elastic-plastic deformation), the energy in the liquid layer can be calculated from equation (8). The following experiments were performed in the same experimental setup but with a backlight and high speed camera were used to record the impact process. The use of this method allowed one to observe the impact from a closer perspective.

Experiments were performed with glass and hardened steel balls (ball bearing) impacting copper and aluminum substrates coated with liquid layers. It was observed that the impaction process resulted in indentation over the substrate. The glass and hardened steel balls however does not undergo plastic deformation which was verified by calculations based on Roger and Reed (1984). Experiments with different liquid viscosities, liquid layer height, substrate material and balls were performed to evaluate Eviscous for varying Stokes and gap Reynolds numbers. Figure 7 shows the schematic of the liquid layer, substrate, and the maximum rebound height.

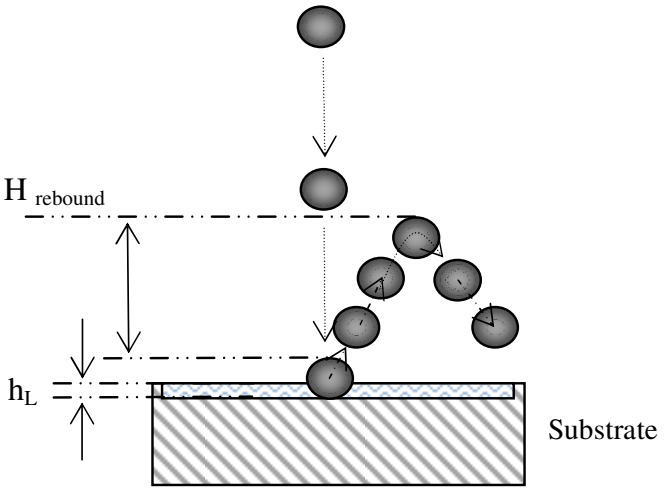

Figure 7. Schematic of the rebound process.

An optical profilometer (Sonsofar PL $\mu$ ) was used to measure the diameter and the depth of the craters formed for plastic indentations. Figure 8 a shows a typical image of the crater formed on a copper substrate and Figure $8 \mathrm{~b}$ shows the cross sectional profile of the crater formed.

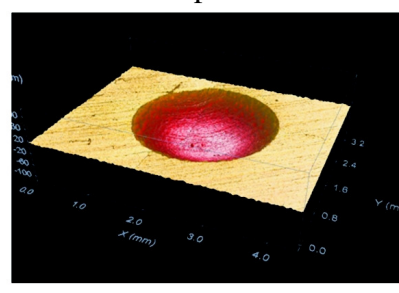

(a)

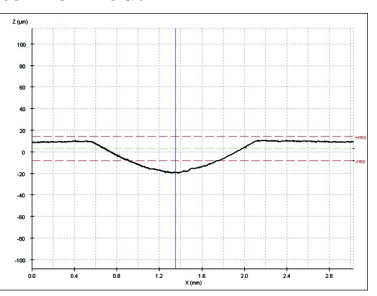

(b)
Figure 8: Crater formed due to particle impaction.

a) Development image of a crater from optical profilometer.

b) Cross sectional view of the crater.

\section{RESULTS (Part2: Energy budget)}

\section{Pure elastic wet collisions}

Figure 9 shows the energy balance for a glass particle impacting a glass substrate coated with liquid film. A priori calculations were done to determine the impact velocity, particle and substrate materials so that only pure elastic collisions occur. The energy that remains at the maximum rebound height $\left(\mathrm{E}_{\mathrm{pot} 2}\right)$ and the viscous energy are plotted as normalized values (normalized by the particle kinetic energy before the collision).

The energy lost in the contact phase is taken as zero as the energy that is expended in elastic deformation is completely imparted to the particle during the rebound phase. It was observed that the particle rebounds for St $>5$. For higher Stokes numbers, the particles rebound but for up to $\mathrm{St}=15$, it was observed that the particles do not travel a distance more than the thickness of the liquid layer.

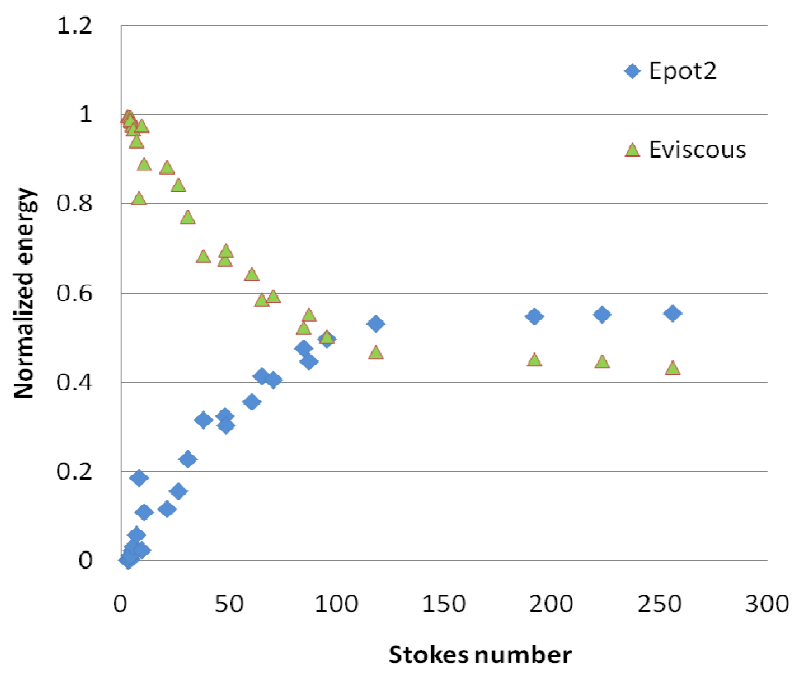

Figure 9. Energy balance for pure elastic wet

For increasing Stokes numbers, the energy dissipated in the viscous layer gradually decreases and for St $>100$, almost $50 \%$ of the particle kinetic energy was dissipated in the viscous layer of the liquid.

\section{Elastic-Plastic wet collisions}

Experiments were performed with glass and hardened steel balls (ball bearing) on copper and aluminum substrates coated with a liquid film. To compare the results with that of elastic collisions, Figure 10 is plotted up to Stokes $=400$. As the viscous dissipation is a calculated value using the measured values of rebound height and the crater volume, any major discrepancy in the calculation of energy expended in crater would lead to a different trend as compared to the pure elastic collision case described in the previous section.

The energy dissipated in the contact phase is calculated using the Rogers and Reeds (1984) model for particles undergoing elastic plastic deformations. This model was basically developed for impaction of micron sized particles and to check for its applicability for the impact of larger particles, dry impaction experiments were performed and crater depth and diameter were compared to that of model predictions and to another empirical model of Thirupatiah and Sundararajan (1990). The indentation dept and crater formation for a hardened steel ball impacting aluminum and 
copper substrate at different velocities and the predictions of the models are shown in figure 11 and 12. The predictions of Roger and Reed model were found to be valid surprisingly well even for larger particles and are used in the present analysis. Further, as the interface surface energy terms are not used to evaluate the energy dissipated in elastic and elastic-plastic deformations, the presence of solid-liquid-solid interface at the impact point does not affect the overall calculations. The details can be found in Van Beek (2001).

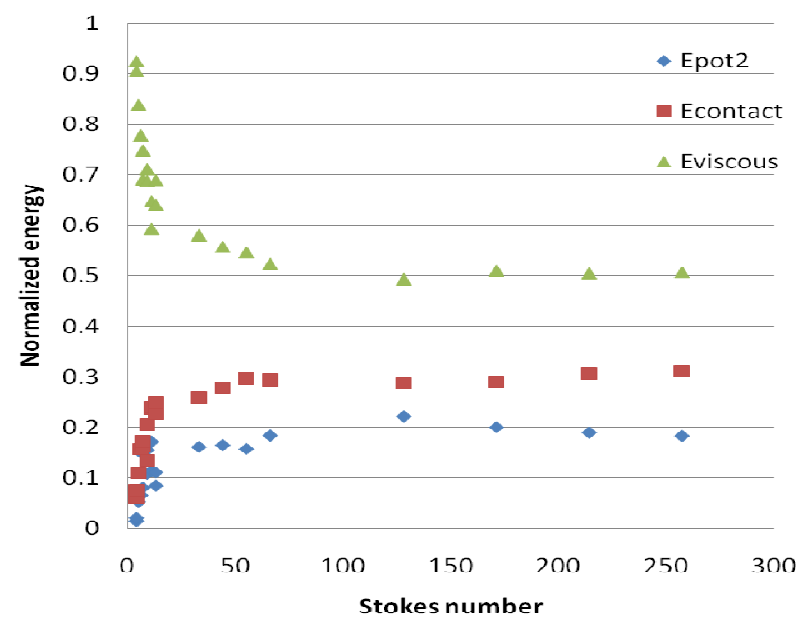

Figure 10. Energy balance for an Elastic-Plastic wet collisions

It can be observed that the energy dissipated in the contact phase of the particle with substrate is higher than the particle energy at the end of rebound phase. Particle rebound was observed for $\mathrm{St}>5$. An interesting observation that can be made is that the energy dissipated in the viscous layer for elastic-plastic deformation follows a similar trend as in a pure elastic collision and for St $>100$, almost $50 \%$ of the initial particle kinetic energy is dissipated in the viscous layer. This in turn also validates the approach for calculating the energy expended in the formation of the crater.

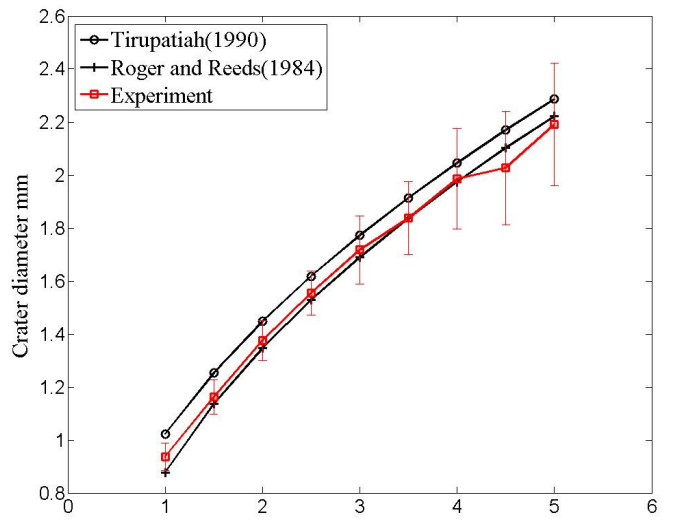

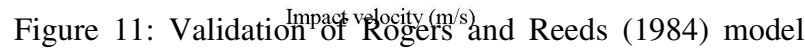
for dry impaction of a hard stainless steel ball over aluminum substrate.

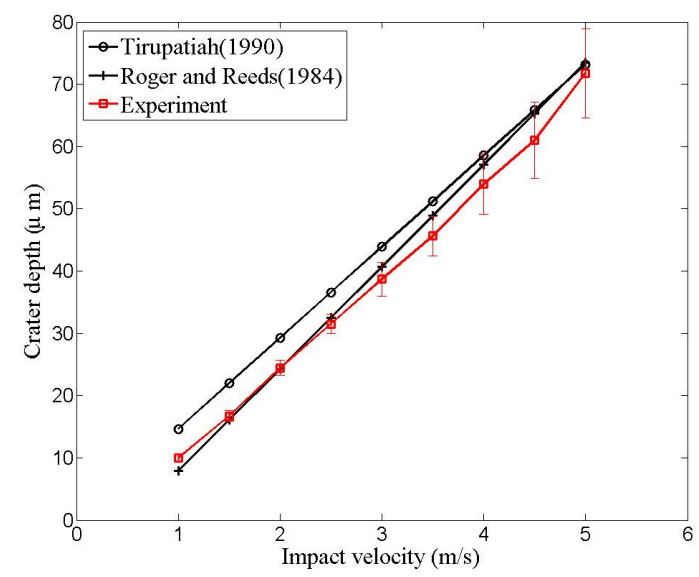

Figure 12: Validation of Rogers and Reeds (1984) models for crater depth formed during a dry impaction of a hard stainless steel ball over aluminum substrate.

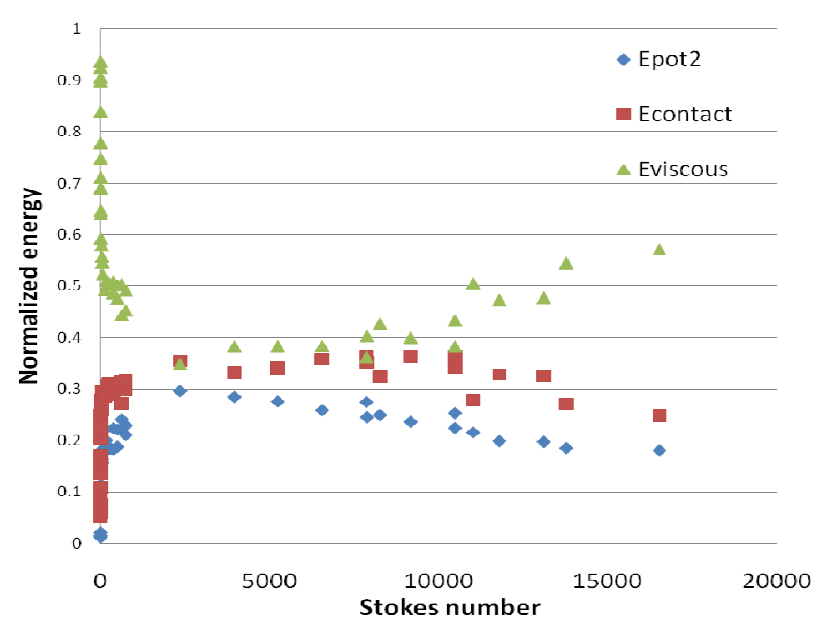

Figure 13: Energy balance for Elastic plastic wet collisions (For St up to 15000)

The energy dissipated in contact phase for higher Stokes numbers displays a trend which is commonly observed in dry impactions implying that the energy lost in the viscous layer reduces for higher Stokes numbers (i.e for higher impact velocities and lower viscosities or a combination of both).

\section{EXPERIMENTS AND RESULTS (Part 3: Controlled fouling)}

Fouling in heat exchangers strongly depend on the humidity in the gas phase and the condensation rates of the vapor species on the heat exchanger surface and the fouled layer (Kaiser et al, 2002). As the thickness of a fouling layer grows, the gas side surface temperature of the layer eventually reaches the same temperature as the gas phase and thus condensation due to the cooling tube stops. In most of the cases involving particulate fouling, the initiation period is a crucial phase and understanding the effect of condensation on the initiation and growth of fouling layers provides vital information to understand and control the overall process. Controlled fouling experiments were 
performed to evaluate the effect of the presence of a liquid layer due to condensation. The details on experimental setup, working and instrumentation can be found in Sathyanarayanarao Subbarao et al (2009). A deposition tube was coated with thin layer of liquid of known viscosity. The liquid coated tube was inserted in test channel in which a stream of gas with seeded particles flow from the top to the bottom. Figure 14 shows the comparison of dry deposition of ash particles and ash particles depositing on a glycerol coated stainless tube. The gas phase velocity (Vg) was maintained at $1.5 \mathrm{~m} / \mathrm{s}$ and the flow was seeded with 0.2 $\mathrm{g} / \mathrm{m}^{3}(\mathrm{Cp})$ of particles. The inlaid images correspond to the processed images to capture the evolution of fouling layer with time. A laser sheet illuminates the region around the deposition tube and snapshots are taken at regular intervals to observe the profile of the fouling layer formed over the cylinder. The thickness of the fouling layer is measured at the center of the cylinder corresponding to the stagnation point. The flow is from the top to the bottom. The dry deposition pattern shows a linear growth from the start of the experiment and flattens after 220 minutes. An asymptotic state is reached after 200 minutes where the layer thickness remains comparatively constant.

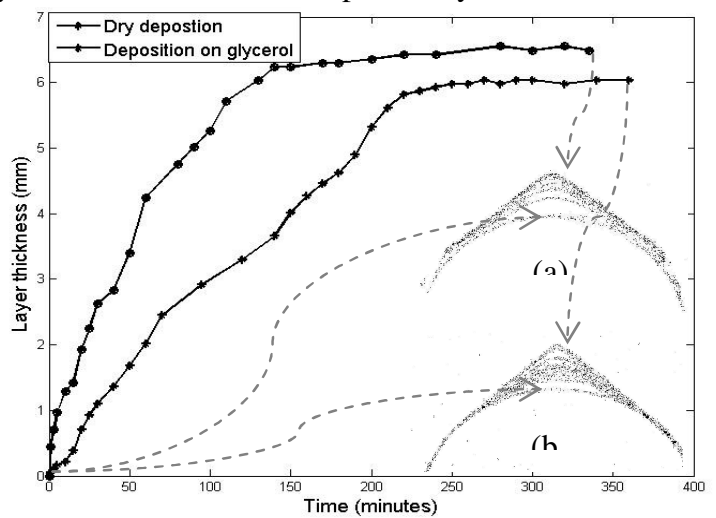

Figure 14 . Ash deposition on a) on glycerol coated tube and $\mathrm{b})$ dry stainless steel tube. $(\mathrm{Vg}=1.5 \mathrm{~m} / \mathrm{s}, \mathrm{Cp}=0.2$ $\left.\mathrm{g} / \mathrm{m}^{3}\right)$

Experiment performed with a liquid layer shows a similar trend in the centre line thickness but the initial rate of growth is much larger than the dry deposition and an asymptotic state is reached much faster. The liquid layer present on the layer captures almost all particles that arrive to the surface. The deposition of initial layer provides more active sites for other particles to deposit and hence more particles deposit over the surface. The circumferential width of layer formed in the wet case is also more and forms a shoulder kind of structure thus changing the geometry of the layer which aides in particle deposition. The effect of liquid layer stops after a while and the layer growth follows a dry deposition pattern. At the end the apex angle formed on the top is similar to the dry deposition.

Figure 15 shows the comparison between glass particles deposition on a dry tube and a glycerol coated tube. Dry deposition showed that glass particles cease to deposit for a gas velocity $\geq 2.5 \mathrm{~m} / \mathrm{s}$. But for the wet deposition, the fouling layer forms even at $3 \mathrm{~m} / \mathrm{s}$. The presence of liquid layer enhances the chances of particle sticking ability as the particle energy is dissipated in the liquid layer. The layer thickness formed for a gas velocity of $3 \mathrm{~m} / \mathrm{s}$ was found to be $0.35 \mathrm{~mm}$ and the profile indicates constant thickness after 50 minutes of operation. The initial growth rate in the presence of liquid layer is however higher than the dry fouling case and the layer growth rate changes abruptly after a thickness of about $0.2 \mathrm{~mm}$ which indicates that the effect of liquid layer is no more important and the deposition is similar to dry deposition. The presence of liquid layer however provides a strong surface for more particles to deposit. A glycerol layer of approximately 40 $70 \mu \mathrm{m}$ was used. The viscosity of glycerol is $1.204 \mathrm{~Pa}$.s and the mean particle diameter was $20 \mu \mathrm{m}$. For these conditions, the Stokes number lies in the range 0.0075 to 0.0262 . Referring back to Figure 9 and 10, it is seen that the particles Stokes number is less than the critical stokes number $(\mathrm{St}=5)$. Thus the particles lose most of their kinetic energy when they reach the cylinder surface and eventually get deposited.

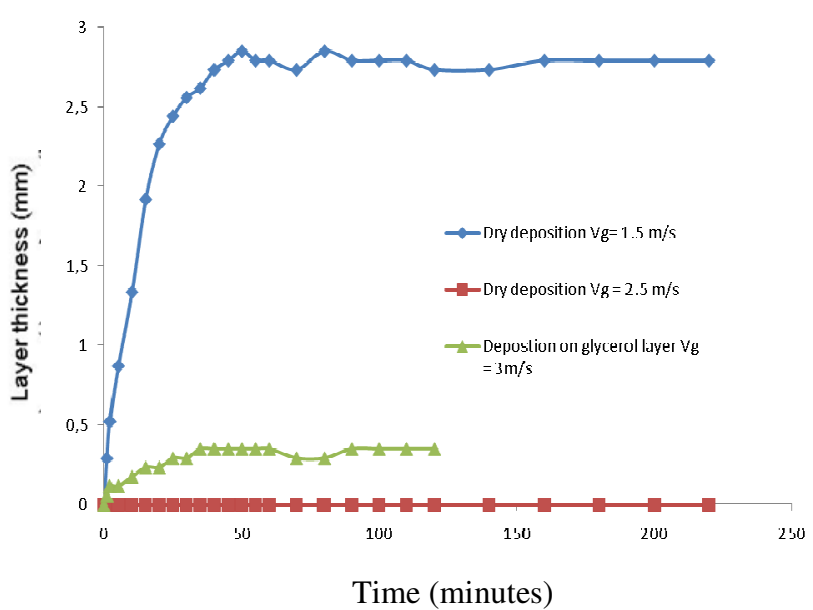

Figure 15. Glass deposition on dry stainless steel tube and on glycerol coated tube. $\left(\mathrm{Cp}=2 \mathrm{~g} / \mathrm{m}^{3}\right)$

Figure 16 shows the Scanning Electron Microscope (SEM) photo of the particles collected from the glass deposition experiment on glycerol coated cylinder. The liquid present on the cylinder surface forms a matrix within which the glass particles deposit. It is also observed that smaller particles adhere on to the larger particles.
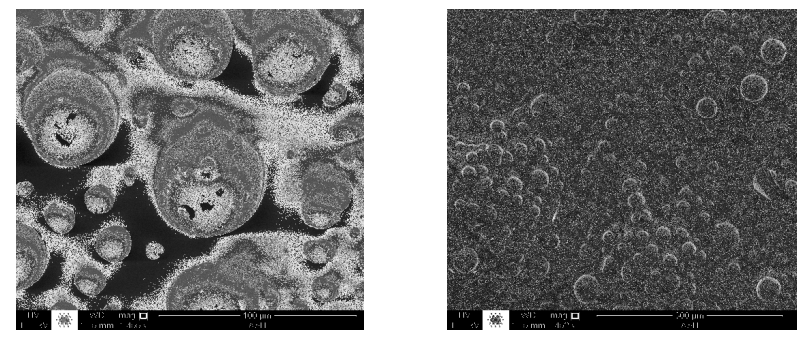

Figure 16. SEM photos of the glass deposited on a cylinder coated with a liquid layer (glycerol). 


\section{CONCLUSIONS:}

Initial stages of condensation of vapors present in the gas phase can be modeled as particles impacting a surface coated with liquid films. A simple model is necessary to define the sticking and removal criteria in the presence of a liquid film. A detailed literature survey was conducted and two models were evaluated with the experimental data and found that the models were limited in their applicability for modeling particulate fouling. Experiments were carried out to evaluate the conditions under which a particle impacting a liquid coated surface sticks. The experimental data provides important information in terms of Stokes numbers. Based on experimental observations, the Sticking criteria for the particle can be based on the model of Davis et al. or it can be safely assumed that a particle will stick if the Stokes number is less than 5. Initial fouling experiments to mimic the initial stages of fouling with condensation effects showed that the presence of the liquid layer enhances the deposition process.

\section{FUTURE WORK:}

A simple model based on wet impaction experiments will be developed to model fouling with condensation effects. Based on the experimental data presented in this article, an empirical formulation will be developed that will provide a condition to determine the amount of energy lost in the liquid layer during a particle impaction process. Based on this, particles can be selected that can be used to remove the previously deposited particles and which can also be used as a parameter to avoid erosion of the heat exchanger surface. Controlled fouling experiments with different particles and liquid layers are planned. The fouling experimental setup will be modified to include the condensation effects by incorporating humidity in the air with different chemical species. The amount of energy dissipated in each phase of the wet impaction will be determined in future which will provide better insight to model removal of particles from a surface with a liquid film.

\section{NOMENCLATURE}

St Stokes number, dimensionless

Re_gap Gap Reynolds number based on liquid layer based on liquid layer thickness, dimensionless

\section{Subscripts}

p particle

o initial or incident

wet wet impaction

c critical

diss dissipation

vis vicous

\section{REFERENCES}

Antonyuk, S, Heinrich S., Deen N. and Kuipers H. (2009), Influence of liquid layers on energy absorbtion during particle impact, Particuology, 7: 245-259.
Bryers, R. W. (1996). Fireside slagging, fouling and high temperature corrosion of heat transfer surface due to impurities in steam rising fuels. Prog. Energy Combustion Sci, 22:29-120.

Davis, R. H. and Serayssol, J. M., (1986). The elastohydrodynamic collision of two spheres, J. Fluid Mechanics, 163:479-497.

Davis, R. H., Rager, D. A. and Good, B. T. (2002), Elastrohydrodynamic rebound of spheres from coated surfaces, J. Fluid Mechanics, 468: 107-119.

Isaak, P., Tran, H., Barham, D. and Reeve, D. (1986), Stickyness of fireside deposits in kraft recovery units. Journal of Pulp and Paper Science, 12(3): 184-192.

Kaiser, S., Antonijevic, D. and Tsotsas, E. (2002), Formation of fouling layers on a heat exchanger element exposed to warm, humid and solid loaded air streams. Experimental thermal and fluid science, 26, 291-297

Rogers, L. N. and Reed, J. (1984), The adhesion of particles undergoing an elastic-plastic impact with a surface, J. Phys.D: Appl Phys, 17, 677-689.

Sathyanarayanarao Subbarao, K. K., Rindt, C. C. M. and van Steenhoven, A. A., 2009, Preliminary study of particulate fouling in a high temperature controlled experimental facility, Heat Exchanger Fouling and Cleaning, Editors: H. Muller-Steinhagen et al., Schladming, Austria, 182-187,

Simons, S. J. R. and Seville, J. P. K. (1994), An analysis of the rupture energy of pendular liquid bridges, Chemical Engineering Science, 14: 2331-2339.

Thirupataiah, Y. and Sundararajan, G. (1990). The volume of crater formed by the impact of a ball against flat target materials - The effect of ball hardness and density. Int. J. Impact Engineering, 9(02):237 - 246.

Tran, H., Mao, X., Kuhn, D., Backman, R., and Hupa, M., (2002), The sticky temperature for recovery boiler fireside deposits. Pulp and Paper Canada, 103(9):29-3.

Vargas, S., Frandsen, F. J. and Dam-Johansen, K., (2001) Rheological properties of high-temperature melts of coal ashes and other silicates. Progress in energy and combustion science, 27:237-429.,

Zenit, R., Hunt, M. L., (1999), Mechanics of immersed particle collisions, Journal of Fluids Engineering, 121: 179184.

Happel, J. and Brenner, H., (1965), Low Reynolds number hydrodynamics, Printice-Hall, New York.

Van Beek, M. C., 2001, Gas-side fouling in heat recovery boilers, $\mathrm{PhD}$ thesis, Eindhoven University of technology.

Drift, A. v. D., (2004), Product gas cooling and ash removal in biomass gasifier, Technical report, ECN-C-04077, ECN Petten, The Netherlands.

Rosner, D. E. and Nagarajan, R., (1992), Inorganic Transformations and Ash Deposition during Combustion, chapter: Deposition dynamics of combustion-generated particles: Summary of recent studies of particle transport mechanisms, capture rates and resulting deposit microstructure/properties, 585-93. Engineering foundation, ASME New York. 